\title{
Platelet-Rich Plasma Injection for Symptomatic Knee Osteoarthritis
}

\author{
Abolfazl Bagherifard ${ }^{1}$; Mahmood Jabalameli ${ }^{1}$; Ali Jahansouz ${ }^{1, *}$; Hooman Yahyazadeh ${ }^{1}$; \\ Hosein Karimi Heris ${ }^{1}$; Mahsa Khezri ${ }^{2}$; Parham Nikraftar $^{2}$; Ramin Bozorgmehr ${ }^{2}$; Tahmineh \\ Mokhtari $^{3}$ \\ ${ }_{2}^{1}$ Bone and Joint Reconstruction Research Center, Shafa Orthopedic Hospital, Iran University of Medical Sciences, Tehran, IR Iran \\ ${ }^{2}$ School of Medicine, Iran University of Medical Sciences, Tehran, IR Iran \\ ${ }^{3}$ Department of Anatomy, School of Medicine, Tehran University of Medical Sciences, Tehran, IR Iran \\ ${ }^{*}$ Corresponding author: Ali Jahansouz, Bone and Joint Reconstruction Research Center, Shafa Orthopedic Hospital, Iran University of Medical Sciences, Tehran, IR Iran. Tel: \\ +98-2133542010, Fax:+98-2133542020, E-mail: a_jahansouz@yahoo.com
}

Received: April 22, 2015; Revised: May 29, 2015; Accepted: June 16, 2015

\begin{abstract}
Background: Platelet-rich plasma (PRP) is plasma of enriched platelets with high concentration of platelet granules and growth factors. The platelet growth factors have a great potential of wound and connective tissue healing used to treat cartilage lesions and retarding the progression of knee osteoarthritis.

Objectives: This study was designed to evaluate PRP injection results in knee osteoarthritis.

Patients and Methods: This was a case-series study of 39 patients and all of them had three injections of PRP for the involved knee (every two weeks) and they were followed up at two weeks, two months and six months post-injection of PRP. All included patients filled the WOMAC (western Ontario and Mcmaster universities arthritis index) standardized and translated to our national language and culture. We analyzed the collected scores of before initiation of PRP injections, the second week, the second month and the sixth month after injections into the knees.

Results: Thirty-nine patients with a mean age of $55.24 \pm 9.27$ years (ranged 40 to 83 ) were evaluated in this study. Six patients (15.4\%) were male and 33 patients (84.6\%) female. There were significant differences in WOMAC score of patients in two months and six months after injection of PRP compared to before injection.

Conclusions: PRP had a great clinical improvement in short-term follow-up for early stages of knee osteoarthritis.
\end{abstract}

Keywords: Arthritis; Platelet- Rich Plasma; Knee; Osteoarthritis

\section{Background}

Osteoarthritis is the leading cause of disability among aged population and its incidence is increased (1). Relevant studies have shown that $10 \%$ of population older than 55 years have severe knee pain due to osteoarthritis (2).

Platelet-rich plasma (PRP) is one of the conservative options for osteoarthritic patients with promising preliminary clinical results $(3,4)$. PRP is a plasma of enriched platelets with high concentrations of platelet granules and growth factors. Platelet derived growth factors have a great potential of connective tissue, wound and vascular repair (5-7). There are so many proteins and over 1500 active proteins stored and identified in platelet alpha granules $(8,9)$.

Some of their important growth factors are fibroblastic growth factor (FGF), platelet derived growth factor (PDGF), vascular endothelial growth factor (VEGF), epithelial growth factor (EGF) and transforming growth factor beta (TGF $\beta$ ). These factors help in matrix protein proliferation and cell development (10-12).

Recently, the use of PRP is increasingly gained attention in sport injuries as a therapeutic tool in orthopedic patients and few basic investigations systematically explored the content of PRP as well as its mechanism (s) of action (13). With increased usage of autologous PRP, various clinical studies reported ambiguous results and questionable efficacy $(14,15)$.

Whether PRP would improve body's natural healing response and facilitate faster rehabilitation, return to play and perhaps less bleeding and pain during recovery, resulted in extensive clinical use of PRP (16).

\section{Objectives}

The current study aimed to investigate the clinical results of PRP injection in osteoarthritic patients according to the western Ontario and Mcmaster universities arthritis index (WOMAC) knee scores after two and six months of intra-articular injection of PRP.

\section{Patients and Methods}

Our research project obtained the project number of 9202-30-24008 from the research administration of Iran university of medical sciences. We selected 60 patients referred

Copyright (c) 2015, Iran University of Medical Sciences. This is an open-access article distributed under the terms of the Creative Commons Attribution-NonCommercial 4.0 International License (http://creativecommons.org/licenses/by-nc/4.0/) which permits copy and redistribute the material just in noncommercial usages, provided the original work is properly cited. 
to our clinic with symptomatic knee osteoarthritis, grades (I, II) of Kellgren-Lawrence classification (17), (Table 1).

The standard radiographic evaluations preoperatively included standing alignment view of both lower extremities, standing lateral X-ray, antero-posterior (AP), patellar view and MRI of involved knee.

\subsection{Inclusion Criteria}

1. Symptomatic osteoarthritis of knee (Kellgren-Lawrence grades 1 - 2).

2. Patients with stable knees, normal tibio-femoral alignment or patellofemoral tracking.

3. Patients with severe pain without improvement of symptoms after conservative treatments.

\subsection{Exclusion Criteria}

1. Inflammatory knee arthritis,

2. Advanced knee osteoarthritis (grades 3 - 4 KellgrenLawrence),

3. Mal-alignment ( $>5$ degree of varus or valgus deformity in coronal plane),

4. History of meniscectomy or meniscal injury proven by MRI.

Among 60 patients, only 39 patients completed the PRP injection courses and follow-up and 39 patients were regularly participated up to the end of our study. All the included patients before injection and at the second week, second month and six months after injection courses completed the WOMAC (western Ontario and McMaster universities arthritis index) score. We used the WOMAC score standardized and translated to our national language and culture (18). All patients participated in this study voluntarily and they filled the consent form.

\subsection{PRP Preparation}

As mentioned earlier, PRP is an autologous concentrate of human platelets in plasma, produced from own patient's centrifuged blood. Production of PRP consists of two steps. In the first step, 8 - $10 \mathrm{~mL}$ of autologous blood aspiration is centrifuged in a double-syringe system and the centrifuge is turned on for five minutes and adjusted to 5,000 rpm (rate per minute, Figure 1). After 5 minutes, RBC (red blood cell) fraction is completely separated from plasma and platelets and WBCs (white blood cells). At the second step, the separated serum is aspirated slowly via the inner syringe from the blood clot and buffy coat layer (Figure 2).

\subsection{Sequences of PRP Injection}

Each of included patients had three injections of PRP by interval of two weeks for the individual knee, in a sterile manner.

\section{Results}

In our study, we observed no major adverse events related to injection and at last 39 patients of 60 persons completed the follow-up. The mean age of patients was 55.24 \pm 9.27 years (ranged 40 to 83 ). Six patients $(15.4 \%$ ) were male and 33 patients (84.6\%) female. Injured knee was the right side in 17 patients (43.6\%) and the left side in 22 patients (56.4\%). All patients had significant improvement in their WOMAC score at 2 and 6 months after injections compared to before injection scores $(\mathrm{P}<0.05)$.

Standardized WOMAC score according to Iranian culture and patients were filled before injection, at the second week, the second month and sixth months after the injections. We got the mentioned score from Mashhad university of medical sciences (18).

No major complications such as joint infection or hematoma were noted. There were significant differences in clinical satisfaction and knee scores at the completion of injections $(\mathrm{P}=0.027)$. There was a significant difference between two months and six months after injections compared to before injections $(\mathrm{P}=0.03, \mathrm{P}=0.021$, respectively), Table 2 .

\begin{tabular}{lr}
\hline Table 1. Kellgren-Lawrence Classification \\
\hline Grade & Description \\
\hline $\mathbf{0}$ & No radiographic features of osteoarthritis \\
$\mathbf{1}$ & Possible joint space narrowing and osteophyte formation \\
$\mathbf{2}$ & Definite osteophyte formation with possible joint space narrowing \\
$\mathbf{3}$ & Multiple osteophytes, definite joint space narrowing, sclerosis and possible bony deformity \\
\hline
\end{tabular}

\begin{tabular}{|c|c|c|c|}
\hline Variables & Minimum & Maximum & $\operatorname{Mean} \pm \mathrm{SD}$ \\
\hline Age & 40 & 83 & $55.24 \pm 9.79$ \\
\hline Before injection & & & $50.77 \pm 13.77$ \\
\hline Two weeks after injection & & & $53.65 \pm 18.24$ \\
\hline Two months after injection & & & $57.74^{\mathrm{a}} \pm 19.51$ \\
\hline Six months after injection & & & $59.54^{\mathrm{a}} \pm 18.69$ \\
\hline
\end{tabular}

\footnotetext{
${ }^{\mathrm{a}}$ A significant difference between two months and six months after injection and before injection times, $(\mathrm{P}=0.03, \mathrm{P}=0.021, \mathrm{respectively})$.
} 


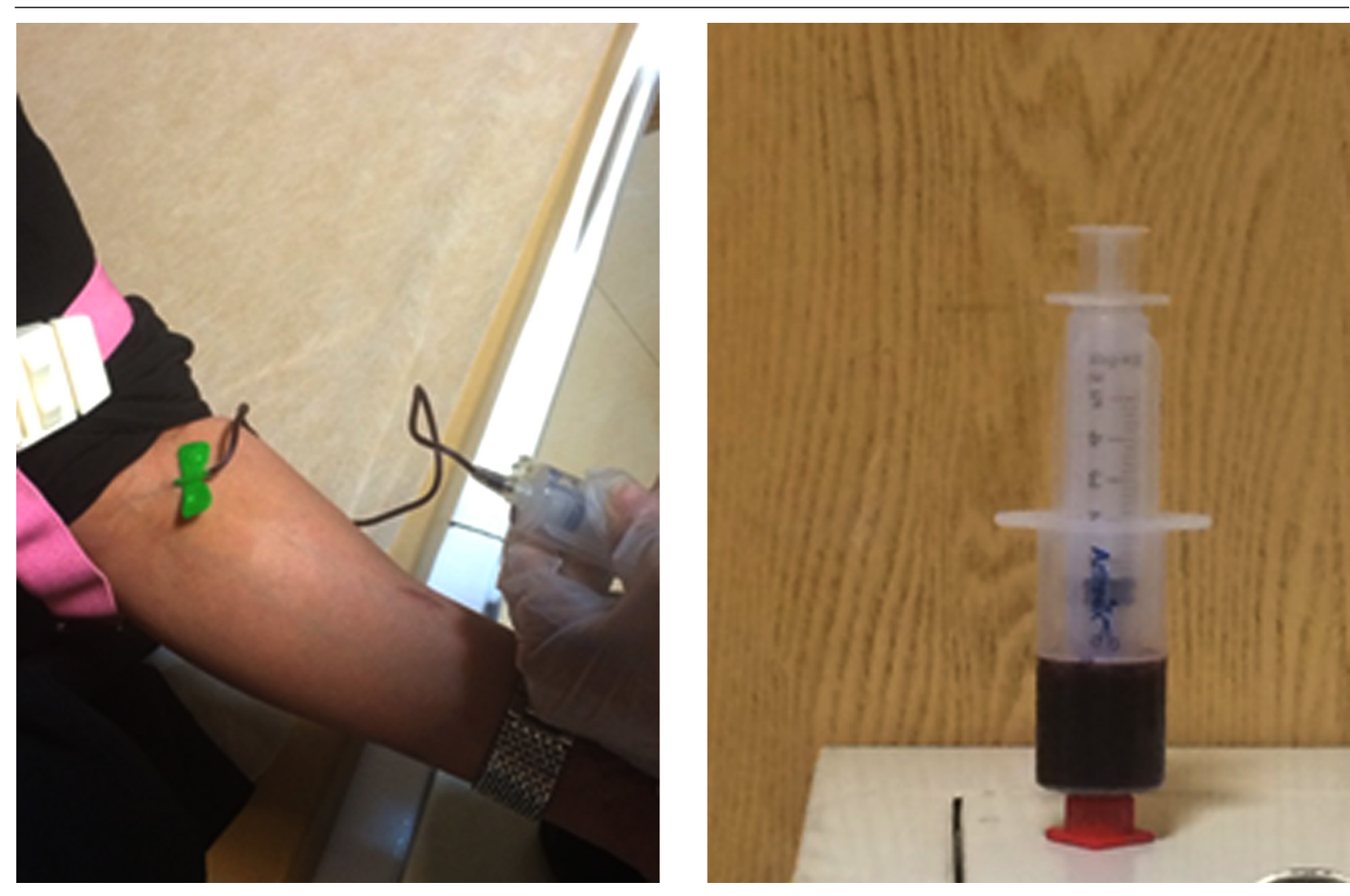

Figure 1. Aspiration of Autologous Blood (8 - 10 mL)

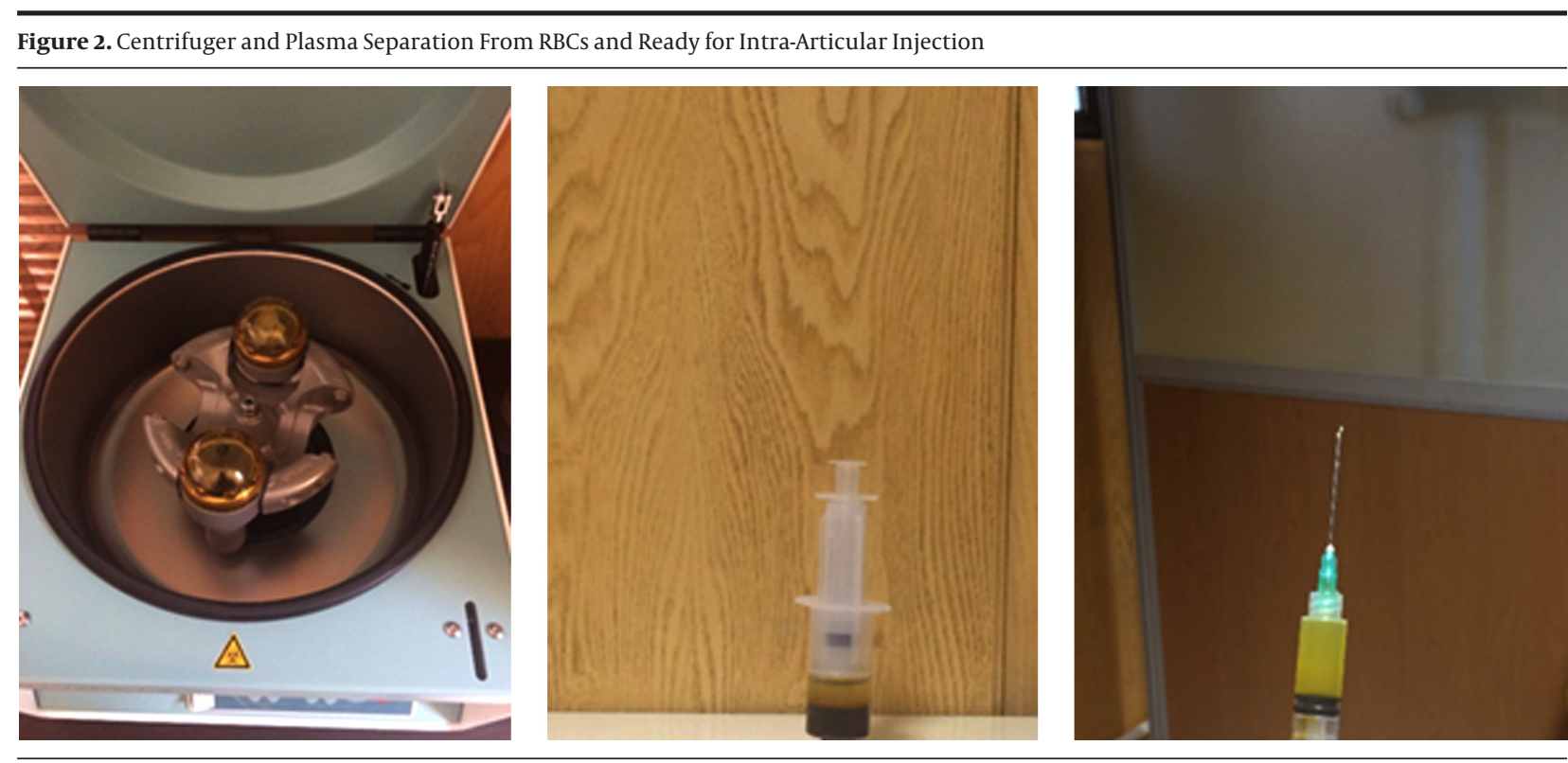

Nearly 3 - 4 mL of PRP is aspirated from (8-10 mL) autologous blood. Total platelet count of PRP was 200,000 per mL.

\section{Discussion}

A number of biological approaches have been proposed to prevent progression of osteoarthritis. The purpose of this study was to investigate the effectiveness of intra- articular PRP injections in active and symptomatic knee osteoarthritis for diminishing pain, improvement in quality of life and activities. 
Now PRP is used in many fields of orthopedic and in the management of tendinopathies, muscular lesions and cartilage damages $(19,20)$. The healing capacity of cartilage makes a challenging problem in knee osteoarthritis treatment (21).

In-vitro studies have shown the effectiveness of PRP in stimulation of chondrocytes in the treatment of osteoarthritis $(22,23)$. Other recent studies documented the effectiveness of platelet growth factors in prevention of joint degeneration by control of synthesis and decreasing extracellular matrix proteins $(24,25)$. Wu et al. in their animal study showed the effectiveness of PRP injections in new cartilage formation (26). Frisbie et al. reported clinical and histological improvement of osteoarthritis in horses (24). Saito et al. in another animal study reported good and promising results in osteoarthritis of rabbit models (25). They reported suppressed progression of osteoarthritis morphologically and histologically in their model. These preventive effects were attributed to stimulation of cartilage matrix metabolism caused by the growth factors contained in PRP.

Nakagawa et al. in their in-vitro study showed the efficacy of autologous PRP in cell proliferation and collagen synthesis of human chondrocytes and suggested a new method of osteoarthritis treatment and use of this method in the treatment of cartilage defects in human (27).

Kon et al. reported positive effect of PRP injection on symptoms and functions of chronic osteoarthritis of knee treated with three injections of PRP, one injection per week. They demonstrated positive effects in about $85 \%$ in scores for patients with a median age less than 60 years but improvement was only $30 \%$ (4).

In another study, authors showed that improvement in clinical results and knee scores deteriorated over 12 to 24 months of follow-up (28). Other authors used intra-articular injections of PRP in knee osteoarthritic patients and showed that PRP combined with proper nutrition, control of body mass index (BMI), exercise and lifestyle can act as a preventive agent in chronic and degenerative diseases (29-31).

All of our patients showed significant improvement at six months follow-up and it is in accordance with the preliminary results of other authors (29-31) with short-term follow-up of PRP injection for knee osteoarthritis.

Anitua et al. in their study on human synovial cells isolated from osteoarthritic patients showed that intraarticular injections of PRP could increase production of hyaluronic acid structure and promote angiogenesis and cell proliferation (32).

In our study, patients with advanced knee osteoarthritis were excluded and they had no associated pathologies such as knee instability, patellofemoral or tibiofemoral severe malalignment, which can affect clinical outcomes. Although in some studies, there have been worse results of PRP injection in female patients (28), but we found no significant differences between men and women results. We found no adverse or major complications during
PRP injection in our patients (such as acute pain or infection or hematoma) and other reports showed its safety without major complications $(4,30,31)$. Intra-articular PRP injections could improve postoperative clinical outcome in these patients. The main limitation of our study was that we did not include a control group. A second limitation was that we followed our patients for six months; long-term follow-up and prospective randomized studies should be performed to clarify PRP effectiveness and durability of clinical improvements. PRP had a therapeutic application with positive and encouraging clinical results in active patients with knee osteoarthritis with mild to moderate grade of knee osteoarthritis.

\section{Authors' Contributions}

Study concept and design: Abolfazl Bagherifard, Mahmood Jabalameli, Ali Jahansouz. Analysis and Interpretation of data: Ali Jahansouz, Hooman Yahyazadeh, Tahmineh Mokhtari. Drafting of the manuscript: Abolfazl Bagherifard, Mahmood Jabalameli, Ali Jahansouz, Mahsa Khezri, Ramin Bozorgmehr, Hosein Karimi Heris, Parham Nikraftar. Critical revision of the manuscript for important intellectual content: Abolfazl Bagherifard, Ali Jahansouz. Study supervision: Abolfazl Bagherifard, Mahmood Jabalameli.

\section{References}

1. Jayabalan P, Hagerty S, Cortazzo MH. The use of platelet-rich plasma for the treatment of osteoarthritis. Phys Sportsmed. 2014;42(3):53-62.

2. Nuki G. Osteoarthritis. In: Luqmani R, Robb J, Porter D editors. Textbook of orthopaedics,Trauma and Rheumatology.. Chicago,IL: Mosby; 2008. p. 193.

3. Anitua E, Sanchez M, Orive G, Andia I. The potential impact of the preparation rich in growth factors (PRGF) in different medical fields. Biomaterials. 2007;28(31):4551-60.

4. Kon E, Buda R, Filardo G, Di Martino A, Timoncini A, Cenacchi A, et al. Platelet-rich plasma: intra-articular knee injections produced favorable results on degenerative cartilage lesions. Knee Surg Sports Traumatol Arthrosc. 2010;18(4):472-9.

5. Nurden AT. Platelets, inflammation and tissue regeneration. Thromb Haemost. 2011;105 Suppl 1:S13-33.

6. Nurden AT, Nurden P, Sanchez M, Andia I, Anitua E. Platelets and wound healing. Front Biosci. 2008;13:3532-48.

7. Pufe T, Petersen WJ, Mentlein R, Tillmann BN. The role of vasculature and angiogenesis for the pathogenesis of degenerative tendons disease. Scand J Med Sci Sports. 2005;15(4):211-22.

8. Broggini N, Hofstetter W, Hunziker E, Bosshardt DD, Bornstein $\mathrm{MM}$, Seto I, et al. The influence of PRP on early bone formation in membrane protected defects. A histological and histomorphometric study in the rabbit calvaria. Clin Implant Dent Relat Res. 2011;13(1):1-12.

9. Borrione P, Gianfrancesco AD, Pereira MT, Pigozzi F. Platelet-rich plasma in muscle healing. Am JPhys Med Rehabil. 2010;89(10):854-61.

10. Wang X, Qiu Y, Triffitt J, Carr A, Xia Z, Sabokbar A. Proliferation and differentiation of human tenocytes in response to platelet rich plasma: an in vitro and in vivo study. J Orthop Res. 2012;30(6):982-90.

11. Klein MB, Pham H, Yalamanchi N, Chang J. Flexor tendon wound healing in vitro: the effect of lactate on tendon cell proliferation and collagen production.J Hand Surg Am. 2001;26(5):847-54.

12. Graziani F, Ivanovski S, Cei S, Ducci F, Tonetti M, Gabriele M. The in vitro effect of different PRP concentrations on osteoblasts and fibroblasts. Clin Oral Implants Res. 2006;17(2):212-9. 


\section{Bagherifard A et al.}

13. Foster TE, Puskas BL, Mandelbaum BR, Gerhardt MB, Rodeo SA Platelet-rich plasma: from basic science to clinical applications. Am J Sports Med. 2009;37(11):2259-72.

14. Redler LH, Thompson SA, Hsu SH, Ahmad CS, Levine WN. Plateletrich plasma therapy: a systematic literature review and evidence for clinical use. Phys Sportsmed. 2011;39(1):42-51.

15. de Vos RJ, van Veldhoven PL, Moen MH, Weir A, Tol JL, Maffulli N. Autologous growth factor injections in chronic tendinopathy: a systematic review. Br Med Bull. 2010;95:63-77.

16. Taylor DW, Petrera M, Hendry M, Theodoropoulos JS. A systematic review of the use of platelet-rich plasma in sports medicine as a new treatment for tendon and ligament injuries. Clin JSport Med. 2011;21(4):344-52.

17. Kellgren JH, Lawrence JS. Radiological assessment of osteo-arthrosis. Ann Rheum Dis. 1957;16(4):494-502.

18. Ebrahimzadeh MH, Makhmalbaf H, Birjandinejad A, Keshtan FG, Hoseini HA, Mazloumi SM. The Western Ontario and McMaster Universities Osteoarthritis Index (WOMAC) in Persian Speaking Patients with Knee Osteoarthritis. Arch Bone Jt Surg. 2014;2(1):57-62.

19. Kon E, Filardo G, Di Martino A, Marcacci M. Platelet-rich plasma (PRP) to treat sports injuries: evidence to support its use. Knee Surg Sports Traumatol Arthrosc. 2011;19(4):516-27.

20. Andia I, Sanchez M, Maffulli N. Platelet rich plasma therapies for sports muscle injuries: any evidence behind clinical practice? Expert Opin Biol Ther. 2011;11(4):509-18.

21. Buckwalter JA, Brown TD. Joint injury, repair, and remodeling: roles in post-traumatic osteoarthritis. Clin Orthop Relat Res. 2004(423):7-16.

22. Sandell LJ, Aigner T. Articular cartilage and changes in arthritis. An introduction: cell biology of osteoarthritis. Arthritis Res. 2001;3(2):107-13.

23. Akeda K, An HS, Okuma M, Attawia M, Miyamoto K, Thonar EJ, et al. Platelet-rich plasma stimulates porcine articular chondrocyte proliferation and matrix biosynthesis. Osteoarthritis Cartilage. 2006;14(12):1272-80.
24. Frisbie DD, Kawcak CE, Werpy NM, Park RD, McIlwraith CW. Clinical, biochemical, and histologic effects of intra-articular administration of autologous conditioned serum in horses with experimentally induced osteoarthritis. Am J Vet Res. 2007;68(3):290-6.

25. Saito M, Takahashi KA, Arai Y, Inoue A, Sakao K, Tonomura H, et al. Intraarticular administration of platelet-rich plasma with biodegradable gelatin hydrogel microspheres prevents osteoarthritis progression in the rabbit knee. Clin Exp Rheumatol. 2009;27(2):201-7.

26. Wu W, Chen F, Liu Y, Ma Q, Mao T. Autologous injectable tissue-engineered cartilage by using platelet-rich plasma: experimental study in a rabbit model. J Oral Maxillofac Surg. 2007;65(10):1951-7.

27. Nakagawa K, Sasho T, Arai M, Kitahara S, Ogino S, Wada Y, et al. P181 Effects of autologous platelet-rich plasma on the metabolism of human articular chondrocytes. Osteoarthritis and Cartilage. 2007;15:B134.

28. Filardo G, Kon E, Buda R, Timoncini A, Di Martino A, Cenacchi A et al. Platelet-rich plasma intra-articular knee injections for the treatment of degenerative cartilage lesions and osteoarthritis. Knee Surg Sports Traumatol Arthrosc. 2011;19(4):528-35.

29. Gobbi A, Bathan L. Biological approaches for cartilage repair. $J$ Knee Surg. 2009;22(1):36-44.

30. Sampson S, Reed M, Silvers H, Meng M, Mandelbaum B. Injection of platelet-rich plasma in patients with primary and secondary knee osteoarthritis: a pilot study. Am J Phys Med Rehabil. 2010;89(12):961-9.

31. Wang-Saegusa A, Cugat R, Ares O, Seijas R, Cusco X, Garcia-Balletbo $\mathrm{M}$. Infiltration of plasma rich in growth factors for osteoarthritis of the knee short-term effects on function and quality of life. Arch Orthop Trauma Surg. 2011;131(3):311-7.

32. Anitua E, Sanchez M, Nurden AT, Zalduendo MM, de la Fuente M, Azofra J, et al. Platelet-released growth factors enhance the secretion of hyaluronic acid and induce hepatocyte growth factor production by synovial fibroblasts from arthritic patients. Rheumatology (Oxford). 2007;46(12):1769-72. 